\title{
The shock index predicts acute mortality outcomes in stroke
}

Stephen J McCall BSc (Hons) ${ }^{\mathrm{a}}$, Stanley D Musgrave MD ${ }^{\mathrm{b}}$, John F Potter DM ${ }^{\mathrm{c}}$, Rachel Hale $\mathrm{BSc}^{\mathrm{d}}$, Allan B Clark PhD ${ }^{\mathrm{e}}$; Mamas A Mamas DPhil ${ }^{\mathrm{f}}$, Anthony K Metcalf $\mathrm{MBChB}^{\mathrm{g}}$, Diana J Day BSc ${ }^{\mathrm{h}}$, Elizabeth A Warburton $\mathrm{PhD}^{\mathrm{i}}$, Max O Bachmann $\mathrm{PhD}^{\mathrm{j}}$, Phyo K Myint $\mathrm{MD}^{\mathrm{k}}$ : On behalf of the Anglia Stroke Clinical Network Evaluation Study (ASCNES) Group

${ }^{a}$ Epidemiology Group, Division of Applied Health Sciences, University of Aberdeen, UK (Permanent address) Nuffield Department of Population Health, University of Oxford, Oxford, UK

This author takes responsibility for all aspects of the reliability and freedom from bias of the data presented and their discussed interpretation

borwich Medical School, University of East Anglia, Norwich, UK.

This author takes responsibility for all aspects of the reliability and freedom from bias of the data presented and their discussed interpretation

'Stroke Research Group, Norfolk \& Norwich University Hospital \& Norwich, UK \& Norwich Medical School, University of East Anglia, Norwich, UK.

This author takes responsibility for all aspects of the reliability and freedom from bias of the data presented and their discussed interpretation

${ }^{\mathrm{d}}$ Norwich Medical School, University of East Anglia, Norwich, UK.

This author takes responsibility for all aspects of the reliability and freedom from bias of the data presented and their discussed interpretation

${ }^{\mathrm{e}}$ Norwich Medical School, University of East Anglia, Norwich, UK.

This author takes responsibility for all aspects of the reliability and freedom from bias of the data presented and their discussed interpretation

${ }^{\mathrm{f}}$ Farr Institute, University of Manchester, Manchester, UK

This author takes responsibility for all aspects of the reliability and freedom from bias of the data presented and their discussed interpretation

${ }^{\mathrm{g}}$ Stroke Research Group, Norfolk \& Norwich University Hospital, Norwich, UK

This author takes responsibility for all aspects of the reliability and freedom from bias of the data presented and their discussed interpretation

${ }^{\text {h}}$ Lewin Stroke \& Rehabilitation Unit, Addenbrooke's Hospital, Cambridge, UK

This author takes responsibility for all aspects of the reliability and freedom from bias of the data presented and their discussed interpretation

'Lewin Stroke \& Rehabilitation Unit, Addenbrooke's Hospital, Cambridge, UK

This author takes responsibility for all aspects of the reliability and freedom from bias of the data presented and their discussed interpretation

${ }^{j}$ Norwich Medical School, University of East Anglia, Norwich, UK

This author takes responsibility for all aspects of the reliability and freedom from bias of the data presented and their discussed interpretation

${ }^{k}$ Epidemiology Group, Division of Applied Health Sciences, University of Aberdeen, UK, AB25 2ZD (Permanent address) \& Norwich Medical School, University of East Anglia, Norwich, UK \& Stroke Research Group, Norfolk \& Norwich University Hospital, Norwich, UK This author takes responsibility for all aspects of the reliability and freedom from bias of the data presented and their discussed interpretation

\section{Address for correspondence}

Professor Phyo Kyaw Myint, Epidemiology Group, Room 4:013 Polwarth Building, Division of Applied Health Sciences, School of Medicine and Dentistry, University of Aberdeen, Foresterhill, Aberdeen, AB25 2ZD, UK. Telephone: 01224437974 and Fax Email:

phyo.myint@abdn.ac.uk

\section{Acknowledgements}

We gratefully acknowledge the funder of this research, NIHR Research for Patient Benefit Programme. We are also indebted to all the research and clinical teams based at the participating NHS Trusts, and the staff from the respective Research \& Development Departments. We also would like to thank the Anglia Stroke \& Heart Clinical Network for their support. 


\section{Funding}

Anglia Stroke Clinical Network Evaluation Study (ASCNES) is funded by the National Institute for Health Research (NIHR), Research for Patient Benefit Programme (PB-PG1208-18240).

\section{Disclaimer}

Anglia Stroke Clinical Network Evaluation Study (ASCNES) is funded by the National Institute for Health Research (NIHR) Research for Patient Benefit Programme (PB-PG-120818240). This paper presents independent research funded by the NIHR under its Research for Patient Benefit (RfPB) programme (Grant Reference Number PB-PG-1208-18240). The views expressed are those of the authors and not necessarily those of the NHS, the NIHR or the Department of Health.

Conflicts of interest: No conflicts of interest to disclose.

Key words: Shock index, prognosis, stroke mortality, acute, systolic blood pressure Abbreviations:

AOR: Adjusted odds ratio

ASCNES: Anglia Stroke Clinical Network Evaluation Study

COPD: Chronic obstructive pulmonary disease

CT: Computed tomography

HR: Heart rate

LACS: Lacunar Stroke

MRI: Magnetic resonance imaging

mRS: Modified Rankin scale

NIHSS: National Institute of Health Stroke Scale

OCSP: Oxfordshire Community Stroke Project

PACS: Partial Anterior Circulation Stroke

POCS: Posterior Circulation Stroke

PVD: Peripheral vascular disease

ROC: Receiver operating characteristic curve

SBP: Systolic blood pressure

SD: Standard Deviation

SI: Shock Index

TACS: Total Anterior Circulation Stroke

TIA: Transient ischemic attacks

\section{Highlights:}

- Both the Shock Index and Systolic BP are good at predicting acute stroke mortality

- SI and SBP are easily available in a clinical setting, and thus useful prognostic markers.

- Shock index is particularly associated with 3-day morality in stroke. 


\begin{abstract}
Background : Shock index (SI) (ratio between heart rate and systolic blood pressure) has been shown to be associated with poor mortality outcomes in trauma and pneumonia; however it has yet to be examined in stroke. We aimed to examine the relationship between SI and acute outcomes of inpatient, 3-day and 7-day mortality in stroke. Secondly, we aimed to compare SI and systolic blood pressure (SBP) alone in predicting above outcomes. Methods: Data from a multicentre prospective cohort study conducted between October 2009 and September 2012 in eight NHS trusts in East of England were analysed. The relationships between SI, SBP and study outcomes were assessed using multivariable logistic regression models using mid-quintile groups as the reference category. Receiver operating characteristic (ROC) curves assessed the discriminating ability between the SI and SBP models.
\end{abstract}

Results: A total of 2121 stroke patients were included (47.4\%men; mean age 77.10 (sd) 12.40) years. The lowest quintile of the SI, had an increased odds of 3-day and 7-day mortality, adjusted odds ratio (AOR) 2.45(95\%CI:1.16-5.17) and 1.88(1.01-3.49), respectively. Patients with the highest quintile of SI also had increased odds of in-patient, 3day and 7-day mortality, AORs 1.85(1.17-2.92), 2.18(1.03-4.63) and 2.45(1.34-4.49), respectively. Similarly, SBP had a U-shape relationship with mortality. All measures had an ROC area under the curve $>0.8$ but there was no difference in the discriminating ability between SI and SBP.

Conclusions: SI at extremely high and low values appeared to predict stroke mortality and appears to be particularly useful in predicting very early (3-day) mortality. 


\subsection{Introduction}

Stroke mortality is highest within first 30 days and both low and high blood pressure during acute stroke phase are associated with poor mortality outcomes. ${ }^{1-3}$ Approximately $80 \%$ of patients have raised blood pressure immediately after a stroke and recent trials have shown that blood pressure lowering has benefits immediately after a stroke. Thus, much effort has been made in examining the association between blood pressure and outcomes following a stroke, while there is a lack of research examining the effects of other physiological parameters of hemodynamics. For example, heart rate is a relatively understudied physiological factor that may be associated with stroke outcome; shown by the contrasting results from two observational studies. ${ }^{4,5}$

The Shock Index (SI) (the ratio between HR and SBP), was used initially to detect hypovolemia in patients with septic or hemorrhagic shock, indicated by a SI value of greater than $0.7 .{ }^{6,7}$ In both these instances it has been a useful prognostic measure and has shown to be associated with poor clinical outcomes. . Whilst a lower SI may have prognostic significance in stroke serving as an indicator for severe neurological stress associated with a high systolic BP and reduced heart rate, large strokes with dysphagia or posterior circulation stroke presenting with vomiting are at high risk of aspiration pneumonia and may be associated with a higher SI as the result of sepsis and/or hypovolemia.

The usefulness of shock index (SI) in predicting acute mortality outcomes in stroke has not been previously examined. In this paper, we examine the relationship between SI at the time of admission and acute outcomes of inpatient, 3 day and 7 day mortality, independently of other prognostic factors. We also examined the relationship between SBP and acute outcomes in stroke. We finally assessed the discriminating ability of both SI and SBP in predicting above outcomes. 


\subsection{Material and Methods}

\subsection{Study Population and design}

The study population was drawn from the Anglia Stroke Clinical Network Evaluation Study (ASCNES). ASCNES's aims, rationale, and study methods have already been previously described. ${ }^{8,9}$ This was a multi-centre prospective cohort study using routinely collected stroke data from eight acute NHS trusts in East of England of all acute stroke admissions during between October 2009 and September 2011, with one year follow up. Only confirmed cases of ischemic and hemorrhagic strokes were eligible to be included in the study thus transient ischemic attacks (TIAs) and other neurological deficits were specifically excluded. Case ascertainment was verified by the patient's medical history, clinical examination and evidence of ischemia or haemorrhage by neuroimaging (computed tomography (CT) or magnetic resonance imaging (MRI)). The study followed the ethical guidelines set out in the Declaration of Helsinki. ${ }^{10}$ Ethical approval was obtained from the Norfolk Research Ethics Committee. The study was funded by the UK National Institute of Health Research, Research for Patient Benefit Programme (PB-PG1208-18240).

We selected variables which have been previously shown to predict mortality outcome and other potential confounders for this outcome in stroke. The three mortality outcomes were death within 3 or 7 days of admission, or at any time during the admission. The prognostic variables used were age, sex, stroke type (ischemic or hemorrhagic), Oxfordshire Community Stroke Project (OCSP) classification based on clinical syndrome regardless of stroke subtype of ischemia or haemorrhage (total anterior stroke (TACS), lacunar stroke (LACS), posterior circulation stroke (POCS) and partial anterior circulation stroke (PACS)), pre-stroke modified Rankin scale (mRS), ${ }^{11,12}$ thrombolysis therapy, heart rate, systolic blood pressure on admission, dates of admission and discharge, and co-morbidities (hypertension, myocardial infarction, diabetes mellitus, hypercholesterolemia, chronic obstructive 
pulmonary disease (COPD), peripheral vascular disease (PVD)) and use of antihypertensive medications on admission (yes/no).

\subsection{Outcomes}

Our primary endpoint was the inpatient mortality and the secondary endpoints were 3-day and 7-day mortality. The 3-day and 7-day mortality outcomes were derived using the date of admission and date of discharge data, thus any patient transferred to another hospital was excluded from the study.

\subsection{Exposures}

The primary predictor variable was the SI at presentation to a secondary care centre, calculated by dividing the heart rate by the systolic blood pressure (SBP). Both SBP and heart rate were measured on the admission by clinical teams from 8 NHS stroke services using the validated BP machines. For the primary analysis the continuous SI was divided into quintiles to better understand the relationship between different level of SI and chosen outcomes. In a secondary analysis we categorized the SI into three categories defined by clinical cut off points, as follows. For clinical cut points we used the normal SI range (0.5-0.7) as the reference category with a SI $<0.5$ as having a low SI score and those with $>0.7$ classed as those with hemodynamic sign of shock. The secondary predictor variable was SBP.

\subsection{Statistical analysis}

Data were analysed using SPSS version 21.0 (Chicago, Illinois, USA). Descriptive statistics such as the Kruskal-Wallis and Chi square tests for trend were used to assess the difference between patient characteristics among the quintiles of the SI and SBP independently. Multiple logistic regression models were constructed for each outcome with the arithmetic quintile groups of SI as the primary exposure variable. For each outcome, model A adjusted for age and sex, model $\mathrm{B}$ adjusted for age, sex and pre-stroke $\mathrm{mRS}$, model $\mathrm{C}$ adjusted for age, 
sex, pre-stroke mRS , OCSP classification and stroke type and model D additionally adjusted for previous co-morbidities, antihypertensive use, and whether the patient was treated for thrombolysis or not. When examining the relationship with SBP only fully adjusted model (Model D) was used for each mortality outcomes. The stratified analysis used the traditionally defined normal values of SI as the exposure for each outcome. We used model D to examine the usefulness of SI stratified by age groups $(<65, \geq 65)$, sex (male and female), OCSP classification (Other than TACS and TACS), prestroke mRS categories (no to moderate disability (mRS $<3)$ and severe prestroke disability $(\mathrm{mRS} \geq 3)$ ) and for ischemic stroke type (due to low numbers for hemorrhagic stroke). The variable of interest was excluded from the each of the respective stratified analyses (e.g. age was not included in the model in age stratified analysis). In all analyses, missing data were excluded from the individual analyses.

Receiver operating characteristic (ROC) curves were used to evaluate the discriminating ability for both SI and SBP for each morality outcomes. The ROC curves used the predicted values from each logistic regression model and a combined analysis tested for equality between each of the curves. We used Stata V.13 (STATA Corp, Texas, USA) for this analysis. 


\subsection{Results}

The sample consisted of 2,625 patients admitted to stroke services of the participating hospitals during the study months; 266 patients were transferred out or did not meet the study inclusion criteria detailed in the methods and therefore were excluded. A further 238 patients were excluded due to missing heart rate or systolic blood pressure data, thus a total of 2,121 patients were eligible. Of them $47.4 \%$ were male, the mean age was 77.10 (sd, 12.40) years, $87.5 \%$ had an ischemic stroke and $42.8 \%$ had a pre-stroke mRS of 0. PACS was the most common OCSP classification (35.2\%) and over half (59.2\%) of the patients had previous history of hypertension. In this study, inpatient mortality was $19.8 \%$ and acute mortality figures were $7.4 \%$ and $11.6 \%$ for 3-day and 7-day mortality, respectively.

Table 1 shows the distribution of sample characteristics across the quintiles of the shock index. There was a higher proportion of ischemic strokes in the fifth quintile in comparison to the first quintile $(91.3 \%$ vs. $85.6, \mathrm{p}<0.03)$. The inpatient mortality was the highest in the fifth quintile at $30.1 \%$ while the other quintiles have a lower proportion of inpatient morality at around 17\%. For both the 3-day and 7-day mortality there appears to be a U/J-shaped relationship according to SI quintiles. In these outcomes the mortality rate appears to be higher at the fifth and first quintiles while the lowest at the third quintile.

Supplementary Table 1 highlights the sample characteristics across the quintiles of SBP. There was a higher proportion of ischemic strokes in the first quintile in comparison to the fifth quintile (90.9\% vs. $80.2 \%$ ). Similar to SI, SBP exhibits a U/J shape relationship with the mortality outcomes. 
Table 2 shows the logistic regression analysis that presents the likelihood of inpatient mortality, 3-day mortality or 7-day mortality. After adjusting for all the covariates, stroke patients who were in the highest SI quintile on admission were at an increased likelihood of inpatient mortality, 3-day mortality and 7-day morality in comparison to stroke patients in the third quintile of the SI (reference category) with the strongest association for inpatient mortality. Patients in the first quintile did not appear to have an increased likelihood of inpatient morality, however these patients had an increased likelihood of 3-day morality with an odds ratio (OR) of 2.45 (95\% CI 1.16-5.17) compared to the third quintile. Similarly stroke patients with low SI had an increased likelihood of a 7-day mortality with an OR of $1.88(95 \% 1.01-3.49)$ compared to the third quintile.

Table 2 also describes the association between SBP and the mortality outcomes using the fully adjusted model (Model D). Higher and lower quintiles of SBP pressure are associated with an increased likelihood of in-patient and 7 day mortality. Unlike SI only high quintiles of SBP are associated with 3 day mortality (OR 2.58 (95\% CI:1.15-5.79)).

Supplementary Table 2 shows the relationship between SI categories defined using lower and upper limit of clinical normal range of SI as the cut off points and the likelihood of in-patient mortality, 3-day mortality and 7-day mortality stratified by age groups ( $<65$ and $\geq 65$ years), sex (males and females), binary OCSP classification (TACS and others) and pre-stroke mRS categories (no to moderate disability $(\mathrm{mRs}<3$ )and $\geq 3)$ ).

Table 3 demonstrates that both SI and SBP are very good predictors of mortality outcomes with excellent area under the curves (AUC) for all of outcomes assessed. SI and SBP had the same AUC for both inpatient mortality and 3-day mortality with an AUC of 0.87 (95\%CI: 
0.85-0.89) and 0.91 (95\% CI: 0.89-0.94), respectively. Both SI and SBP had a similarly high AUC for 7 day mortality. However, there was no significant difference between SI and SBP in any of the mortality outcomes with a $\mathrm{p}$ value of $>0.05$ for each combined ROC curve analysis. 


\subsection{Discussion}

To our knowledge, this is the first study to examine the association between the shock index and acute mortality outcomes in stroke. We have found that both low and high values of the SI are associated with increased risk of 3-day and 7-day mortality, while only high values of the SI are associated with inpatient mortality. We have further shown that low and high levels of SBP are associated with inpatient and 7-day mortality. Both SI and SBP are very good predictors of morality outcomes, however there is little differentiation between the discriminating ability. Only low levels of SI are associated with 3-day mortality. Thus SI may be a useful indicator of very early mortality outcome in acute stroke.

\subsection{Context of findings}

A high SI has been reported to have a prognostic significance in conditions such as sepsis and severe volume depletion. ${ }^{6,13,14}$ Previous research has shown that stroke patients with pneumonia and stroke associated infection are at an increased risk of mortality. ${ }^{15,16}$ Thus, an explanation for a higher likelihood of mortality in the high SI group may be due to stroke associated infections. To our knowledge, this is the first study to show the predictive value of the higher shock index in stroke patients.

Previous studies have used the SI to assess outcomes in patients with community acquired pneumonia, acute circulatory failure and predicting patients that require immediate intensive therapy. ${ }^{13,14,17,18}$ Moreover, a recent study that used prospectively collected audit data showed that a SI of $>1.0$ was predictive of inpatient mortality in individuals age 90 years and over. ${ }^{19}$ In this report, for the first time we have shown that low values of the SI were associated with very acute stroke outcomes; this indicates that mortality was higher among patients with signs of a low SI than those with a normal SI. A possible explanation for this is 
that a low SI is only apparent in severe stroke injuries indicating severe insult to the brain. One possible explanation for this is the Cushing's reflex, this is where there is a sharp rise in intracranial pressure (ICP) which attempts to maintain cerebral perfusion with resultant reflex bradycardia. Kalmer et al. ${ }^{20}$ showed the Cushing reflex indicated by hypertension and bradycardia predicted poor intracranial perfusion and high ICP even during the neurological endoscopy, a minimally invasive procedure used in the management of brain tumors and tumor related hydrocephalus. Another study highlighted that mortality after a subarachnoid hemorrhage was predicted by high ICP, which demonstrated poor intracranial perfusion.

${ }^{21}$ However, further work is required to assess whether there is a biologically plausible relationship between the Cushing's reflex and acute stroke mortality. This will however require serial measurements of both heart rate and blood pressure.

Previous research has shown that both high and low SBP measurements after stroke are associated with morality. ${ }^{2,22,23}$ These studies showed that there was a U-shape relationship between systolic blood pressure and acute mortality outcome. The high levels of mortality in the fifth quintile could be explained by the high proportion of hemorrhagic strokes and previous history of hypertension seen in this sample. Previous research has suggested this association may be due an increased likelihood of a fatal cerebral edema in high SBP patients. ${ }^{24,25}$ The association between low SBP (first quintile) and mortality may be due to inadequacy of cerebral perfusion due to low mean arterial pressure and subsequent reduce cerebral blood flow. ${ }^{2}$

\subsection{Strengths of the study}

Our study is likely to be representative of the stroke population of the UK and western populations as it used a systematic sampling strategy over an adequately long time period 
from eight different NHS trusts. As this is an observational study thus causation between the SI and poor stroke outcomes cannot be drawn. Nevertheless, the SI may be useful in providing prognostic information as well as in identifying those patients with stroke who are at highest risk of mortality outcomes.

\subsection{Limitations}

Our study has a few limitations. Although the study population is generalizable to the UK it may not reflect the case mix of other world regions due to the lack of ethnic diversity in this study population; the study population is mainly Caucasians (>95\%). However, there is no reason to believe that other ethnic populations would have different physiological responses in stroke with regard to BP and HR. In the UK setting, NIHSS (National Institute of Health Stroke Scale) which is an acute stroke severity marker was not assessed routinely for all strokes. Therefore, we have NIHSS data available only in those who were assessed for thrombolysis treatment. However, we were able to control for OCSP classification which usually reflects the NIHSS. As with all quantitative studies missing values are an issue, missing values in the pre-stroke mRS reduced the size of the final model. Nevertheless, this may only have reduced the study power and only likely to attenuate the results. Whilst assessment of multiple outcomes may increase the risk of Type 1 errors, arguably prognostic information on very early mortality in stroke is very important clinically as this information can potentially guide the clinicians in making treatment decisions including appropriate level and place of care.

\subsection{Conclusion}

In summary, our study illustrated that SI and SBP are significant predictor of acute stoke morality. SI is a very simple index derived from two readily available vital signs at the time of stroke admission making it useful for the clinical setting. Given that the SI is an index, it is 
likely to be more user friendly in clinical setting compared to continuous BP values. This paper uniquely highlights that patients at the extremes of the SI are at an increased likelihood

of poor acute stroke mortality outcomes. Our results suggest that at low SI predicts very early mortality in stroke (3-day mortality) whilst high SI may be more useful beyond 3 days where complications and sepsis (e.g. post stroke pneumonia) are prevalent. While both high and low values of SBP were predictive of poor acute outcomes, SI has additional benefit of predicting 3-day mortality. Further work should be replicated to support the findings of this study and should also examine whether incorporating SI in prognostic scoring systems would improve the clinical prediction rules in stroke.

\subsection{References}

(1) Kwok CS, Skinner J, Metcalf AK, Potter JF, Myint PK. Prior antiplatelet or anticoagulant therapy and mortality in stroke. Heart . 2012;98:712-717.

(2) Leonardi-Bee J, Bath PMW, Phillips SJ, Sandercock PAG, for the IST Collaborative Group. Blood Pressure and Clinical Outcomes in the International Stroke Trial. Stroke . 2002;33:1315-1320.

(3) Willmot M, Leonardi-Bee J, Bath PMW. High Blood Pressure in Acute Stroke and Subsequent Outcome A Systematic Review. Hypertension . 2004;43:18-24.

(4) Tomii Y, Toyoda K, Suzuki R, Naganuma M, Fujinami J, Yokota C, et al. Effects of 24Hour Blood Pressure and Heart Rate Recorded With Ambulatory Blood Pressure Monitoring on Recovery From Acute Ischemic Stroke. Stroke . 2011;42:3511-3517.

(5) Geeganage C, Tracy M, England T, Sare G, Moulin T, Woimant F, et al. Relationship Between Baseline Blood Pressure Parameters (Including Mean Pressure, Pulse Pressure, and 
Variability) and Early Outcome After Stroke: Data From the Tinzaparin in Acute Ischaemic Stroke Trial (TAIST). Stroke . 2011;42:491-493.

(6) Berger T, Green J, Horeczko T, Hagar Y, Garg N, Suarez A, et al. Shock index and early recognition of sepsis in the Emergency Department: Pilot study. Western Journal of Emergency Medicine . 2013;14:168-174.

(7) Allgöwer M, Burri C. "Shock index". Dtsch Med Wochenschr . 1967;92:1947-1950.

(8) Kwok CS, Musgrave SD, Price GM, Dalton G, Myint PK. Similarity of patient characteristics and outcomes in consecutive data collection on stroke admissions over one month compared to longer periods. Acta Vet Scand . 2014:342.

(9) Myint PK, Potter JF, Price GM, Barton GR, Metcalf AK, Hale R, et al. Evaluation of stroke services in Anglia stroke clinical network to examine the variation in acute services and stroke outcomes. BMC Health Services Research . 2011;11.

(10) World Medical Association. World Medical Association Declaration of Helsinki. Ethical principles for medical research involving human subjects. Bull World Health Organ . 2001;79:373-374.

(11) Kwok CS, Potter JF, Dalton G, George A, Metcalf AK, Ngeh J, et al. The SOAR Stroke Score Predicts Inpatient and 7-Day Mortality in Acute Stroke. Stroke . 2013;44:2010-2012.

(12) Kwok CS, Clark A, Ford GA, Durairaj R, Dixit AK, Davis J, et al. Association between prestroke disability and inpatient mortality and length of acute hospital stay after acute stroke. J Am Geriatr Soc . 2012;60:726-732. 
(13) Berger T, Green J, Horeczko T, Hagar Y, Garg N, Suarez A, et al. Shock index and early recognition of sepsis in the Emergency Department: Pilot study. Western Journal of Emergency Medicine . 2013;14:168-174.

(14) Sankaran P, Kamath AV, Tariq SM, Ruffell H, Smith AC, Prentice P, et al. Are Shock Index and Adjusted Shock Index useful in predicting mortality and length of stay in community-acquired pneumonia? Eur J Intern Med . 2011;22:282-285.

(15) Katzan IL, Cebul RD, Husak SH, Dawson NV, Baker DW. The effect of pneumonia on mortality among patients hospitalized for acute stroke. Neurology . 2003;60:620-625.

(16) Kwan J, Pickering RM, Kunkel D, Fitton C, Jenkinson D, Perry VH, et al. Impact of stroke-associated infection on long-term survival: a cohort study. Journal of Neurology, Neurosurgery \& Psychiatry . 2013;84:297-304.

(17) Rady MY, Smithline HA, Blake H, Nowak R, Rivers E. A Comparison of the Shock Index and Conventional Vital Signs to Identify Acute, Critical Illness in the Emergency Department. Ann Emerg Med . 1994;24:685-690.

(18) Rady MY, Nightingale P, Little RA, Edwards JD. Shock index: A re-evaluation in acute circulatory failure. Resuscitation . 1992;23:227-234.

(19) Wilson AH, Kidd AC, Skinner J, Musonda P, Pai Y, Lunt CJ, et al. A simple 5-point scoring system, NaURSE (Na+, Urea, Respiratory Rate and Shock Index in the Elderly), predicts in-hospital mortality in oldest old. Age and Ageing . 2014;43:352-357.

(20) Kalmar AF, Van Aken J, Caemaert J, Mortier EP, Struys MMRF. Value of Cushing reflex as warning sign for brain ischaemia during neuroendoscopy. British Journal of Anaesthesia . 2005;94:791-799. 
(21) Ryttlefors M, Howells T, Nilsson P, Ronne-Engström E, Enblad P. Secondary insults in subarachnoid hemorrhage: Occurrence and impact on outcome and clinical deterioration. Neurosurgery . 2007;61:704-714.

(22) Robinson TG, Potter JF. Blood pressure in acute stroke. Age Ageing . 2004;33:6-12.

(23) Castillo J, Leira R, García MM, Serena J, Blanco M, Dávalos A. Blood Pressure Decrease during the Acute Phase of Ischemic Stroke Is Associated with Brain Injury and Poor Stroke Outcome. Stroke . 2004;35:520-527.

(24) Willmot M, Leonardi-Bee J, Bath PMW. High Blood Pressure in Acute Stroke and Subsequent Outcome A Systematic Review. Hypertension . 2004;43:18-24.

(25) Spence JD. New treatment options for hypertension during acute ischemic or hemorrhagic stroke. Current Treatment Options in Cardiovascular Medicine . 2007;9:242246. 
Table 1: Sample characteristics of 2121 stroke patients in ASCNES (2009-2011) by quintiles of the Shock Index

\begin{tabular}{|c|c|c|c|c|c|c|c|}
\hline & & \multicolumn{5}{|c|}{ Quintiles of Shock Index } & \\
\hline & & Q1 & Q2 & Q3 & Q4 & Q5 & \\
\hline \multicolumn{2}{|c|}{ Shock Index (SI) } & $0-0.396$ & $0.397-0.467$ & $0.468-0.542$ & $0.543-0.643$ & $0.645-1.5$ & \\
\hline \multicolumn{2}{|c|}{ SI (n) } & 424 & 423 & 426 & 423 & 425 & P-value \\
\hline \multicolumn{2}{|c|}{ Age (Mean) } & 78 & 78 & 76 & 77 & 77 & 0.12 \\
\hline \multicolumn{2}{|c|}{ Sex (\% Male $)$} & $208(49.1)$ & $217(51.3)$ & $197(46.4)$ & $201(47.5)$ & $182(42.8)$ & 0.03 \\
\hline \multicolumn{2}{|c|}{ Systolic Bp (Mean) } & 185 & 167 & 156 & 146 & 131 & $<0.0001$ \\
\hline \multicolumn{2}{|c|}{ Length of stay in days (mean) } & 15 & 15 & 17 & 16 & 18 & 0.14 \\
\hline \multicolumn{2}{|c|}{ Heart rate (mean) } & 63 & 72 & 79 & 86 & 102 & $<0.0001$ \\
\hline \multirow{6}{*}{ pre-stroke modified Rankin Scale (\%) } & 0 & $196(54.6)$ & $198(54.8)$ & $181(49.5)$ & $182(51.4)$ & $151(43.3)$ & \multirow{6}{*}{$<0.0001$} \\
\hline & 1 & $71(19.8)$ & $74(20.5)$ & $62(16.9)$ & $58(16.4)$ & $54(15.5)$ & \\
\hline & 2 & $30(8.4)$ & $31(8.6)$ & $47(12.8)$ & $46(13.0)$ & $33(9.5)$ & \\
\hline & 3 & $31(8.6)$ & $32(8.9)$ & $45(12.3)$ & $43(12.1)$ & $44(12.6)$ & \\
\hline & 4 & $20(5.6)$ & $17(4.7)$ & $23(6.3)$ & $15(4.2)$ & $50(14.3)$ & \\
\hline & 5 & $11(3.1)$ & $9(2.5)$ & $8(2.2)$ & $10(2.8)$ & $17(4.9)$ & \\
\hline Stroke type (\%) & Ischemic & $352(85.6)$ & $348(84.5)$ & $359(87.3)$ & $368(88.7)$ & $369(91.3)$ & 0.003 \\
\hline \multirow{2}{*}{ Oxford Stroke Project Classification (\%) } & $\mathrm{LACS}^{\mathrm{a}}$ & $93(21.9)$ & $102(24.1)$ & $95(22.3)$ & $94(22.2)$ & $84(19.8)$ & \multirow{2}{*}{0.08} \\
\hline & $\mathrm{PACS}^{\mathrm{b}}$ & $151(35.6)$ & $148(35.0)$ & $147(34.5)$ & $160(37.8)$ & $140(32.9)$ & \\
\hline
\end{tabular}




\begin{tabular}{|c|c|c|c|c|c|c|c|}
\hline & $\mathrm{POCS}^{\mathrm{c}}$ & $55(13.0)$ & $63(14.9)$ & $48(11.3)$ & $56(13.2)$ & $43(10.1)$ & \\
\hline & $\mathrm{TACS}^{\mathrm{d}}$ & $87(20.0)$ & $63(14.9)$ & $94(22.1)$ & $68(16.1)$ & $109(25.6)$ & \\
\hline & Undetermined & $40(9.4)$ & $47(11.1)$ & $42(9.9)$ & $45(10.6)$ & $49(11.5)$ & \\
\hline & Treat for thrombolysis - No (\%) & $402(94.8)$ & $403(95.3)$ & $403(94.6)$ & $403(95.3)$ & $408(96.0)$ & 0.47 \\
\hline \multirow{7}{*}{ Pre-co-morbidities (Yes \%) } & Myocardial Infarction & $112(26.4)$ & $105(24.8)$ & $96(22.5)$ & $95(22.5)$ & $134(31.5)$ & 0.24 \\
\hline & Peripheral Vascular disease & $13(3.1)$ & $21(5.0)$ & $13(3.1)$ & $15(3.5)$ & $21(4.9)$ & 0.43 \\
\hline & Diabetes Mellitus & $75(17.7)$ & $72(17.0)$ & $66(15.5)$ & $70(16.5)$ & $85(20.0)$ & 0.50 \\
\hline & Hypertension & $273(64.4)$ & $265(62.6)$ & $236(55.4)$ & $244(57.7)$ & $238(56.0)$ & 0.004 \\
\hline & Antihypertensive Rx & $228(53.8)$ & $221(52.2)$ & 207 (48.6) & $222(52.5)$ & $214(50.4)$ & 0.59 \\
\hline & Hypercholesterolemia & $68(16.0)$ & $81(19.1)$ & $71(16.7)$ & $75(17.7)$ & $72(16.9)$ & 0.95 \\
\hline & COPD & $12(2.8)$ & $23(5.4)$ & $27(6.3)$ & $30(7.1)$ & $37(8.7)$ & $<0.0001$ \\
\hline \multirow{3}{*}{ Outcomes } & In patient mortality $n=419$ & $73(17.2)$ & $70(16.5)$ & $74(17.4)$ & $74(17.5)$ & $128(30.1)$ & $<0.0001$ \\
\hline & 3 Day mortality $n=158$ & $35(8.3)$ & $22(5.2)$ & $25(5.9)$ & $29(6.9)$ & $47(11.1)$ & 0.07 \\
\hline & 7 day mortality $n=245$ & $49(11.6)$ & $43(10.2)$ & $34(8.0)$ & $45(10.6)$ & $74(17.4)$ & 0.01 \\
\hline
\end{tabular}

${ }^{\mathrm{a}}$ Lacunar Stroke; ${ }^{\mathrm{b}}$ Partial Anterior Circulation Stroke; ${ }^{\mathrm{C}}$ Posterior Circulation Stroke; ${ }^{\mathrm{d}}$ Total Anterior Circulation Stroke 
Table 2: Odds ratios of in-patient mortality, 3-day mortality and 7 day mortality in each of the quintiles of the Shock Index in ASCNES: logistic regression models

\begin{tabular}{|c|c|c|c|c|c|c|c|c|c|}
\hline & \multicolumn{2}{|r|}{ Q1 } & \multicolumn{2}{|r|}{ Q2 } & \multirow{2}{*}{$\begin{array}{c}\text { Q3 } \\
\text { Reference }\end{array}$} & \multicolumn{2}{|r|}{$\mathrm{Q} 4$} & \multicolumn{2}{|c|}{ Q5 } \\
\hline Outcomes and models & OR & 95\% CI & OR & 95\% CI & & OR & 95\% CI & OR & 95\% CI \\
\hline In-hospital mortality $^{\mathrm{e}}$ & & & & & & & & & \\
\hline $\mathrm{A}^{\mathrm{a}}$ & 0.94 & $(0.65-1.35)$ & 0.93 & $(0.65-1.35)$ & 1 & 1.01 & $(0.70-1.46)$ & 2.1 & $(1.50-2.94)$ \\
\hline $\mathrm{B}^{\mathrm{b}}$ & 0.97 & $(0.64-1.46)$ & 0.9 & $(0.59-1.36)$ & 1 & 0.91 & $(0.60-1.38)$ & 1.59 & $(1.08-2.35)$ \\
\hline $\mathrm{C}^{\mathrm{c}}$ & 1.02 & $(0.63-1.64)$ & 1.1 & $(0.68-1.78)$ & 1 & 1.25 & $(0.77-2.02)$ & $1.89^{* * *}$ & $(1.20-2.97)$ \\
\hline $\mathrm{D}^{\mathrm{d}}$ & 1.02 & $(0.63-1.66)$ & 1.09 & $(0.67-1.78)$ & 1 & 1.25 & $(0.77-2.03)$ & $1.85^{* * *}$ & $(1.17-2.92)$ \\
\hline 3-day mortality ${ }^{\mathrm{e}}$ & & & & & & & & & \\
\hline $\mathrm{A}^{\mathrm{a}}$ & 1.45 & $(0.85-2.49)$ & 0.90 & $(0.50-1.63)$ & 1 & 1.22 & $(0.70-2.14)$ & $2.07 * *$ & $(1.24-3.46)$ \\
\hline $\mathrm{B}^{\mathrm{b}}$ & $1.74 *$ & $(0.93-3.23)$ & 0.84 & $(0.41-1.73)$ & 1 & 1.21 & $(0.62-2.34)$ & 1.67 & $(0.91-3.08)$ \\
\hline $\mathrm{C}^{\mathrm{c}}$ & $2.27 *$ & $(1.09-4.74)$ & 1.04 & $(0.45-2.41)$ & 1 & 1.89 & $(0.86-4.15)$ & $2.32 *$ & $(0.86-4.15)$ \\
\hline $\mathrm{D}^{\mathrm{d}}$ & $2.45 *$ & $(1.16-5.17)$ & 1.1 & $(0.47-2.58)$ & 1 & 1.9 & $(0.86-4.19)$ & $2.18^{*}$ & $(1.03-4.63)$ \\
\hline 7-day mortality ${ }^{\mathrm{e}}$ & & & & & & & & & \\
\hline $\mathrm{A}^{\mathrm{a}}$ & $1.49 *$ & $(0.94-2.38)$ & 1.33 & $(0.82-2.14)$ & 1 & 1.41 & $(0.88-2.26)$ & $2.5^{* * * *}$ & $(1.61-3.88)$ \\
\hline $\mathrm{B}^{\mathrm{b}}$ & 1.56 & $(0.91-2.65)$ & 1.33 & $(0.77-2.30)$ & 1 & 1.38 & $(0.80-2.38)$ & $1.86^{* * *}$ & $(1.11-3.11)$ \\
\hline
\end{tabular}




\begin{tabular}{|c|c|c|c|c|c|c|c|c|c|}
\hline $\mathrm{C}^{\mathrm{c}}$ & 1.84 & $(0.99-3.41)$ & 1.75 & $(0.93-3.30)$ & 1 & $2.14 *$ & $(1.14-4.03)$ & $2.48 * *$ & $(1.36-4.54)$ \\
\hline $\mathrm{D}^{\mathrm{d}}$ & $1.88 *$ & $(1.01-3.49)$ & 1.76 & $(0.93-3.35)$ & 1 & $2.11 *$ & $(1.12-3.99)$ & $2.45 * *$ & $(1.34-4.49)$ \\
\hline Inpatient mortality ${ }^{\mathrm{f}, \mathrm{d}}$ & $1.97 *$ & $(1.19-3.26)$ & 1.64 & $(0.99-2.73)$ & 1 & 1.23 & $(0.73-2.06)$ & $2.02 * * *$ & $(1.22-3.33)$ \\
\hline 3 Day mortality ${ }^{\mathrm{f}, \mathrm{d}}$ & 1.84 & $(0.79-4.28)$ & 0.82 & $(0.32-2.09)$ & 1 & 1.5 & $(0.65-3.48)$ & $2.58 *$ & $(1.15-5.79)$ \\
\hline 7 Day mortality ${ }^{\mathrm{f}, \mathrm{d}}$ & $2.09 *$ & $(1.09-4.02)$ & 1.00 & $(0.49-2.02)$ & 1 & 1.63 & $(0.85-3.14)$ & $2.23 *$ & $(1.18-4.24)$ \\
\hline
\end{tabular}

$*<0.05 * * * * \leq 0.01 * * * \leq 0.001 .{ }^{e}$ Shock Index quintiles (Q1 (<0.396), Q2 (0.397-0.467), Q3 (0.468-0.542), Q4 (0.543-0.6430 and Q5 (0.645-1.50)).

${ }^{\mathrm{f}}$ Systolic blood pressure quintiles (Q1 (<132 mmHg), Q2 (132-148mmHg), Q3 (149-162mmHg), Q4 (163-182mmHg), Q5 (=>183mmHg)

${ }^{\mathrm{a}}$ Model A age and sex adjusted ( $\left.\mathrm{n}=2116\right) ;{ }^{\mathrm{b}}$ Model B= Model A + mRS ( $\left.\mathrm{n}=1787\right) ;{ }^{\mathrm{c}}$ Model C= Model B + Oxford Stroke Project Classification + Stroke Type $(\mathrm{n}=1752) ;{ }^{\mathrm{d}}$ Model D =Model C + Previous Medical History +treated for thrombolysis $(\mathrm{n}=1752)$. 
Table 3. The comparison of the receiver operating characteristic (ROC) curve analysis, examining the difference in the discriminating ability between quintiles of the shock index and systolic blood pressure.

\begin{tabular}{|c|c|c|c|c|c|}
\hline & & $\begin{array}{c}\text { Area under ROC } \\
\text { curve }\end{array}$ & $95 \% \mathrm{CI}$ & P-value & $\chi^{2}$ Value \\
\hline Inpatient & Shock Index model & 0.87 & $(0.84-0.89)$ & \multirow{2}{*}{0.85} & \multirow{2}{*}{0.04} \\
\hline mortality & Systolic blood pressure model & 0.87 & $(0.85-0.89)$ & & \\
\hline 3-day & Shock Index model & 0.91 & $(0.89-0.94)$ & \multirow{2}{*}{0.63} & \multirow{2}{*}{0.23} \\
\hline mortality & Systolic blood pressure model & 0.91 & $(0.89-0.94)$ & & \\
\hline 7-day & Shock Index model & 0.86 & $(0.83-0.89)$ & \multirow{2}{*}{0.26} & \multirow{2}{*}{0.61} \\
\hline mortality & Systolic blood pressure model & 0.87 & $(0.84-0.90)$ & & \\
\hline
\end{tabular}


Supplementary Table 1: Sample characteristics of 2,121 stroke patients in ASCNES (2009-2011) by quintiles of the systolic blood pressure

\begin{tabular}{|c|c|c|c|c|c|c|c|}
\hline & & \multicolumn{5}{|c|}{ Quintiles of systolic blood pressure } & \\
\hline & & Q1 & Q2 & Q3 & Q4 & Q5 & \\
\hline \multirow{2}{*}{\multicolumn{2}{|c|}{ Quintiles (mmHg) }} & $<131$ & $132-148$ & $149-162$ & $163-182$ & $\geq 163$ & \\
\hline & & $425(20.0)$ & $449(21.2)$ & $416(19.6)$ & $419(19.8)$ & $412(19.4)$ & P-value \\
\hline \multicolumn{2}{|c|}{ Age (Mean) } & 76 & 76 & 78 & 78 & 78 & 0.122 \\
\hline \multicolumn{2}{|c|}{ Sex (\% Male) } & $209(49.2)$ & $207(46.2)$ & $216(51.9)$ & $191(45.6)$ & $182(44.2)$ & 0.175 \\
\hline \multicolumn{2}{|c|}{ LOS (mean) } & 16 & 17 & 16 & 15 & 14 & 0.074 \\
\hline \multicolumn{2}{|c|}{ Heart rate (mean) } & 81 & 80 & 81 & 80 & 81 & 0.746 \\
\hline \multirow{6}{*}{ pre-stroke modified Rankin Score $(\%)$} & 0 & $149(42.8)$ & $216(56.5)$ & $181(50.8)$ & $176(49.3)$ & $186(53.8)$ & \multirow{6}{*}{0.007} \\
\hline & 1 & $58(16.7)$ & $58(15.2)$ & $76(21.3)$ & $64(17.9)$ & $63(18.2)$ & \\
\hline & 2 & $38(10.9)$ & $38(9.9)$ & $33(9.3)$ & $48(13.4)$ & $30(8.7)$ & \\
\hline & 3 & $47(13.5)$ & $39(10.2)$ & $39(11.0)$ & $38(10.6)$ & $32(9.2)$ & \\
\hline & 4 & $39(11.2)$ & $21(5.5)$ & $22(6.2)$ & $20(5.6)$ & $23(6.6)$ & \\
\hline & 5 & $17(4.9)$ & $10(2.6)$ & $5(1.4)$ & $11(3.1)$ & $12(3.5)$ & \\
\hline Stroke type (\%) & Ischaemic & $368(90.9)$ & $401(91.8)$ & $356(88.6)$ & 351 (85.6) & $320(80.2)$ & $\leq 0.001$ \\
\hline \multirow{3}{*}{ Oxford Stroke Project Classification (\%) } & $\mathrm{LACS}^{\mathrm{a}}$ & $98(23.1)$ & $95(21.2)$ & $93(22.4)$ & $95(22.7)$ & $87(21.1)$ & \multirow{3}{*}{0.921} \\
\hline & PACS $^{b}$ & $124(29.2)$ & $174(38.8)$ & 169 (40.6) & 149 (35.6) & $130(31.6)$ & \\
\hline & $\mathrm{POCS}^{\mathrm{c}}$ & $56(13.2)$ & $50(11.1)$ & $54(13.0)$ & $52(12.4)$ & $53(12.9)$ & \\
\hline
\end{tabular}




\begin{tabular}{|c|c|c|c|c|c|c|c|}
\hline & TACS $^{\mathrm{d}}$ & $97(22.8)$ & $85(18.9)$ & $62(14.9)$ & $78(18.6)$ & $97(23.5)$ & \\
\hline & Undetermined & $50(11.8)$ & $45(10.0)$ & $38(9.1)$ & $45(10.7)$ & $45(10.9)$ & \\
\hline & Treat for thrombolysis - Yes (\%) & $27(6.4)$ & $24(5.3)$ & $21(5.0)$ & $19(4.5)$ & $11(2.7)$ & 0.014 \\
\hline \multirow{7}{*}{ Pre-co-morbidities (Yes \%) } & Myocardial Infarction & $138(32.5)$ & $110(24.5)$ & $107(25.7)$ & $107(25.5)$ & $80(19.4)$ & $\leq 0.001$ \\
\hline & Peripheral Vascular disease & $23(5.4)$ & $14(3.1)$ & $18(4.3)$ & $13(3.1)$ & $15(3.6)$ & 0.240 \\
\hline & Diabetes Mellitus & $89(20.9)$ & $57(12.7)$ & $69(16.6)$ & $73(17.4)$ & $80(19.4)$ & 0.754 \\
\hline & Hypertension & $242(56.9)$ & $248(55.2)$ & $228(54.8)$ & $277(66.1)$ & $261(63.3)$ & 0.002 \\
\hline & Antihypertensive Rx & $221(52.0)$ & $226(50.3)$ & $205(49.3)$ & $235(26.1)$ & $205(49.8)$ & 0.857 \\
\hline & Hypercholesterolemia & $70(16.5)$ & $91(20.3)$ & $70(16.8)$ & $69(16.5)$ & $67(16.3)$ & 0.471 \\
\hline & COPD & $28(6.6)$ & $37(8.2)$ & $22(5.3)$ & $29(6.9)$ & $13(3.2)$ & 0.026 \\
\hline \multirow{3}{*}{ Outcomes } & In patient mortality $n=419$ & $108(25.4)$ & $82(18.3)$ & $52(12.5)$ & $73(17.4)$ & $104(25.2)$ & 0.818 \\
\hline & 3 Day mortality $n=158$ & $44(10.4)$ & $21(4.7)$ & $17(4.1)$ & $28(6.7)$ & $48(11.7)$ & 0.261 \\
\hline & 7 day mortality $n=245$ & $69(16.2)$ & $35(7.8)$ & $25(6.0)$ & $47(11.2)$ & $69(16.7)$ & 0.374 \\
\hline
\end{tabular}

${ }^{\mathrm{a}}$ Lacunar Stroke; ${ }^{\mathrm{b}}$ Partial Anterior Circulation Stroke; ${ }^{\mathrm{c} P o s t e r i o r ~ C i r c u l a t i o n ~ S t r o k e ; ~}{ }^{\mathrm{d}}$ Total Anterior Circulation Stroke 
Supplementary Table 2: Odds Ratios of in-patient mortality, 3-day mortality and 7-day mortality using normal range values of Shock Index as cut off points in stratified analyses by age categories, sex, stroke classification, prestroke disability and in ischemic stroke subtype.

\begin{tabular}{|c|c|c|c|c|c|c|c|}
\hline & & & $<0.5$ & $95 \% \mathrm{CI}$ & $0.5-0.69$ & $>0.7$ & $95 \% \mathrm{CI}$ \\
\hline \multirow{6}{*}{ Age } & \multirow{2}{*}{ In patient mortality } & $<65$ & 0.26 & $0.04-1.85$ & 1 & 5.52 & $(0.83-36.61)$ \\
\hline & & $\geq 65$ & 0.99 & $(0.70-1.39)$ & 1 & $1.96^{* * * *}$ & $(1.24-3.11)$ \\
\hline & \multirow{2}{*}{3 Day mortality } & $<65$ & 0.75 & $\begin{array}{l}(0.01-40.35) \\
\end{array}$ & 1 & 0.27 & $\begin{array}{l}(0.00-27.89) \\
\end{array}$ \\
\hline & & $\geq 65$ & 1.22 & $(0.70-2.12)$ & 1 & $2.46 * *$ & $(1.2-5.02)$ \\
\hline & \multirow{2}{*}{7 Day mortality } & $<65$ & .750 & $(0.08-7.42)$ & 1 & 5.69 & $(0.26-126.56)$ \\
\hline & & $\geq 65$ & 1.05 & $(0.68-1.61)$ & 1 & 1.74 & $(0.98-3.08)$ \\
\hline \multirow{7}{*}{ Sex } & \multirow{3}{*}{ In patient mortality } & & & & & & \\
\hline & & Female & 1.1 & $(0.71-1.72$ & 1 & $2.35 * *$ & $(1.30-4.28)$ \\
\hline & & Male & 0.76 & $(0.45-1.29)$ & 1 & $2.14 *$ & $(1.09-4.19)$ \\
\hline & \multirow{2}{*}{3 Day mortality } & Female & 1.16 & $(0.58-2.33)$ & 1 & 2.18 & $(0.84-5.63)$ \\
\hline & & Male & 1.45 & $(0.60-3.52)$ & 1 & 2.94 & $(1.00-8.68)$ \\
\hline & \multirow{2}{*}{7 Day mortality } & Female & 1.39 & $(0.80-2.43)$ & 1 & $2.56^{*}$ & $(1.23-5.34)$ \\
\hline & & Male & 0.78 & $(0.40-1.54)$ & 1 & 1.48 & $(0.61-3.58)$ \\
\hline & & & & & & & \\
\hline \multirow{4}{*}{ Oxford Stroke Project Classification } & \multirow{2}{*}{ In patient mortality } & Other type & 0.87 & $(0.57-1.34)$ & 1 & $2.21 * *$ & $(1.27-3.82)$ \\
\hline & & TACS & 1.21 & $(0.70-2.07)$ & 1 & $2.34 *$ & $(1.13-4.83)$ \\
\hline & \multirow{2}{*}{3 Day mortality } & Other type & 0.92 & $(0.41-2.06)$ & 1 & 2.8 & $(0.98-8.02)$ \\
\hline & & TACS & 1.66 & $(0.79-3.47)$ & 1 & 2.46 & $(1.00-6.08)$ \\
\hline
\end{tabular}




\begin{tabular}{|c|c|c|c|c|c|c|c|}
\hline & \multirow{2}{*}{7 Day mortality } & Other type & 0.93 & $(0.51-1.69)$ & 1 & $2.74 *$ & $(1.29-5.81)$ \\
\hline & & TACS & 1.29 & $(0.71-2.32)$ & 1 & 1.45 & $(0.68-3.10)$ \\
\hline \multirow{6}{*}{ Pre-stroke mRS } & \multirow{2}{*}{ In patient mortality } & $<3$ & 1.06 & $(0.70-1.61)$ & 1 & $3.27 * * *$ & $(1.85-5.78)$ \\
\hline & & $\geq \mathbf{3}$ & 0.75 & $(0.44-1.26)$ & 1 & 1.4 & $(0.77-2.53)$ \\
\hline & \multirow{2}{*}{3 Day mortality } & $<3$ & 1.81 & $(0.91-3.61)$ & 1 & $2.73 * *$ & $(1.01-7.38)$ \\
\hline & & $\geq \mathbf{3}$ & 0.6 & $(0.28-1.30)$ & 1 & 1.39 & $(0.63-3.04)$ \\
\hline & \multirow{2}{*}{7 Day mortality } & $<3$ & 1.18 & $(0.71-1.95)$ & 1 & 1.92 & $(0.92-3.99)$ \\
\hline & & $\geq 3$ & 0.89 & $(0.47-1.71)$ & 1 & $2.10 *$ & $(1.05-4.18)$ \\
\hline \multirow{3}{*}{ Stroke Type - Ischemi } & \multicolumn{2}{|c|}{ In patient mortality } & 0.84 & $(0.57-1.23)$ & 1 & $1.94 * *$ & $(1.21-3.11)$ \\
\hline & \multicolumn{2}{|c|}{3 Day mortality } & 0.87 & $(0.41-1.84)$ & 1 & 206 & $(0.92-4.58)$ \\
\hline & \multicolumn{2}{|c|}{7 Day mortality } & 0.77 & $(0.46-1.30)$ & 1 & 1.58 & $(0.86-2.88)$ \\
\hline
\end{tabular}

$*<0.05 * * \leq 0.01 * * * \leq 0.001$. Models adjusted for age, sex, mRS, Oxford Stroke Project Classification, Stroke Type, previous co-morbidities, treated for thrombolysis. ${ }^{\text {a Only }}$ included Ischemic strokes in analysis. 